\title{
Évaluation de l'impact de l'assainissement urbain sur la qualité des eaux du bassin versant de la rivière Chaudière à l'aide du système de modélisation intégrée GIBSI
}

\section{Evaluation of the impact of a municipal clean water program on water quality of the Chaudière river watershed using the integrated modelling system GIBSI}

\author{
A. Mailhot, A. N. Rousseau, E. Salvano, R. Turcotte et J. P. Villeneuve
}

Volume 15, numéro hors-série, 2002

URI : https://id.erudit.org/iderudit/705491ar

DOI : https://doi.org/10.7202/705491ar

\section{Aller au sommaire du numéro}

\section{Éditeur(s)}

Université du Québec - INRS-Eau, Terre et Environnement (INRS-ETE)

\section{ISSN}

0992-7158 (imprimé)

1718-8598 (numérique)

\section{Découvrir la revue}

Citer cet article

Mailhot, A., Rousseau, A. N., Salvano, E., Turcotte, R. \& Villeneuve, J. P. (2002). Évaluation de l'impact de l'assainissement urbain sur la qualité des eaux du bassin versant de la rivière Chaudière à l'aide du système de modélisation intégrée GIBSI. Revue des sciences de l'eau / Journal of Water Science, 15, 149-172. https://doi.org/10.7202/705491ar

\section{Résumé de l'article}

Le programme d'assainissement des eaux de Québec (PAEQ), mis en place à la fin des années 1970, s'est d'abord attaqué au problème de la pollution ponctuelle d'origine urbaine. Plusieurs stations de traitement des eaux usées municipales ont été construites dans le cadre de ce programme réduisant de façon importante les charges de polluants d'origine urbaine. La question demeure toutefois de savoir dans quelle mesure les charges urbaines rejetées avant et l'après l'instauration de ce programme peuvent entraîner des dépassements de différents critères de l'eau. La présente étude a pour objectif d'examiner cette problématique pour le bassin versant de la rivière Chaudière en utilisant le système de modélisation intégrée GIBSI (Gestion Intégrée par Bassin versant à l'aide d'un Système Informatisé). Deux scénarios d'assainissement urbain ont été examinés, l'un représentatif de la période avant la mise en place du programme, le début des années 1980, et un autre représentatif de la période plus récente. Deux chroniques météorologiques ont été utilisées (années 1983 et 1994). L'estimation des probabilités de dépassement de différents critères de l'eau montre une nette réduction de ces probabilités après mise en place du programme. Pour la $\mathrm{DBO}_{5}$ et l'azote total, les charges urbaines rejetées actuellement n'entraînent pas de dépassement des critères de qualité de l'eau pour les deux années retenues. Toutefois, les résultats montrent que les charges en phosphore total d'origine urbaine peuvent à elles seules entraîner des probabilités de dépassement importantes lors d'étiage. 


\title{
Évaluation de l'impact de l'assainissement urbain sur la qualité des eaux du bassin versant de la rivière Chaudière à l'aide du système de modélisation intégrée GIBSI
}

\section{Evaluation of the impact of a municipal clean water program on water quality of the Chaudière river watershed using the integrated modelling system GIBSI}

\author{
A. MAILHOT ${ }^{1}{ }^{*}$, A.N. ROUSSEAU ${ }^{1}$, E. SALVANO ${ }^{1}$, R. TURCOTTE ${ }^{2}$, \\ J.-P. VILLENEUVE 1
}

SUMMARY

In 1978, the Québec Government put in place a provincial municipal clean water program, referred to as the Programme d'assainissement des eaux du Québec (PAEQ), to restore the province's rivers to their natural state. The program focussed primarily on the problem of municipal waste loads and, hence, a large number of wastewater treatment plants (WWTP) were constructed during the 1980's and the 1990's. At the beginning of the 1980's, only a few percent of the population had their waste water treated, but this figure had increased to over 95\% by 1997 (figure 1). In the Chaudière river watershed, this program resulted in the construction of more than 35 WWTP over an 18-year period. Although an impressive effort was devoted to reduce municipal waste loads, a question remains: how does this reduction translate into terms of overall improvement of water quality at the watershed level?

The Chaudière river watershed was considered for this application. This watershed covers an area of $6682 \mathrm{~km}^{2}$ and is located south of Quebec City. Land use is dominated by forest (62\%) followed by agricultural land (33\%), urban area $(3.6 \%)$, and water $(1.7 \%)$. The total population in 1996 was 173129 and was mainly located in the northern part (i.e., the downstream region) of the watershed. A total of $\mathbf{4 4}$ municipal point loads were identified (see figure 2 and table 1), most of them corresponding to WWTP. Characteristic data on municipal WWTP for year 1995 were obtained from the Quebec Department of the Environment (Ministère de l'Environnement et de la Faune du Québec, 1997) and used to estimate average concentrations of total phosphorus and the biological oxygen demand (BOD). Data included affluent and effluent concentrations at various WWTP. Since no data were available

1. INRS-Eau, 2800 rue Einstein, CP 7500 , Sainte-Foy, Québec, Canada, G1V 4C7.

2. Ministère de l'Environnement, 675 boul. René-Lévesque Est, Aile René-Lévesque, $2^{\mathrm{e}}$ étage, boîte 28 , Québec (Québec), G1R 5 V7.

* Correspondance. E-mail : Alain_Mailhot@inrs-ete.uquebec.ca

** Les commentaires seront reçus jusqu'au 30 avril 2003. 
for nitrogen, a concentration of $40 \mathrm{mg}-\mathrm{N} / \mathrm{L}$ was used (TCHOBANOGLOUS and SCHOEDER, 1985; NOVOTNY and CHESTERS, 1981). Nitrogen removal efficiency for different types of treatment was estimated from available data compiled by the Environment ministry of Quebec (table 2). An average, per capita, daily wastewater volume of $0.73 \mathrm{~m}^{3}$ was derived. This large value indicates a large infiltration capacity in the sewer network and, on average, a poor structural state.

Results were analysed at four locations distributed along the river. These points corresponded to the locations of the four major water quality monitoring stations (figure 3). Total annual loads within the sub-watersheds defined by these points were estimated (table 3). Affluent characteristics were considered for the simulation of the pre-PAEQ period. Figure 4 represents the evolution of total phosphorus loads at station 2340012 (water intake for the town of Charny, the most downstream point). It shows that an important reduction occurred in 1986. For the same sub-watershed, the overall reduction for the 1982-1999 period was $38 \%$ for total phosphorus, 37\% for total nitrogen and $83 \%$ for $B O D$.

Assessment of the impact of the PAEQ was done using the integrated modelling system GIBSI ('Gestion intégrée par bassin versant à l'aide d'un système informatisé") (ROUSSEAU et al., 2000; MAILHOT et al., 1997; VILLENEUVE et al., 1998). Only pollutant loads originating from sewer networks were considered; pollutant loads from industrial plants not connected to a municipal sewer network were not considered in this study. Similarly, diffuse sources of pollution from urban area or agricultural land were not taken into account. Two scenarios were considered: a first scenario associated with the 1983 year corresponding to the pre-PAEQ period; and a second scenario associated with the 1994 year describing the post-PAEQ period (following load reductions). Two hydrological reference years were also selected, namely, years 1983 and 1994. The former corresponded to a significantly drier year than the latter. At station 2340012 (Charny water intake), this difference was even more pronounced when considering that the cumulative summer flow was 6.9 times larger in 1994 than in 1983 . Four simulations corresponding to different combinations of representative municipal waste loads and meteorological conditions were performed (table 5).

Simulation results at the four control points were compared with water quality standards (WQS) for total phosphorus $(0.03 \mathrm{mg}-P / \mathrm{L}$, aesthetic WQS for prevention of eutrophication in rivers) and $\mathrm{BOD}(3 \mathrm{mg}-\mathrm{BOD} / \mathrm{L}$, sanitary WQS for domestic use of water requiring disinfection treatment only). The probabilities of exceeding these WQS were defined (equation 1) as the number of days (daily computational time step) where simulated values exceeded a given value (complementary cumulative distribution function). Table 8 introduces annual probabilities of exceeding WQS at the four control points and for the different simulations whereas table 9 introduces those for the summer period. At station 2340012 (Charny water intake), for hydrological year 1994, a comparison of pre- and post-PAEQ conditions for total phosphorus shows that post-PAEQ condition lowered the number of days exceeding WQS by 49. Similar reductions were reached at the other control points. For the 1994 summer period, the reduction was not as pronounced since the probability of exceeding WQS was 0.24 for pre-PAEQ situation and it decreased to 0.21 for the post-PAEQ condition. These probabilities worsened under the 1983 summer conditions as concentrations exceeded the WQS $98 \%$ of the time at station 2340014.

Results for BOD were quite different. They showed that probabilities of exceeding WQS were null for both years following reduction of BOD loads. The worst case before the PAEQ load reduction program occurred when using the 1983 meteorological data at station 2340012 (Charny's water uptake) with a 0.22 probability of exceeding the criterion on an annual basis. 
On a summer basis, the highest probability of exceeding WQS was observed at station 2340014 with a value of 0.54 .

The WQS for nitrate-nitrite nitrogen $(10 \mathrm{mg}-\mathrm{N} / \mathrm{t})$ was used because there is no criterion for total nitrogen. The largest value of total nitrogen obtained for the four simulations was $3.96 \mathrm{mg}$ - $\mathrm{N} / \mathrm{l}$, a value significantly smaller than the criterion for nitrite-nitrate nitrogen only. The median value of nitrogen concentrations for pre-PAEQ simulations was $0.86 \mathrm{mg}-\mathrm{N} / \mathrm{l}$ and for postPAEQ simulations $0.54 \mathrm{mg}$ - $\mathrm{N} / \mathrm{l}$. These results clearly showed that urban loads of nitrogen are unlikely to lead to water quality problems even under prePAEQ conditions.

The major conclusions of this work are: (1) nitrogen loads from urban origin were unlikely to produce concentrations exceeding WQS under either post- or pre-PAEQ conditions, (2) BOD concentrations were smaller than WQS for the whole period covered by the two simulation years, and (3) phosphorus loads from urban origin were responsible for high probabilities of exceeding WQS.

Key-words: urban point loads, exceedance probability, QUAL2E, municipal clean water program.

\section{RÉSUMÉ}

Le programme d'assainissement des eaux de Québec (PAEQ), mis en place à la fin des années 1970, s'est d'abord attaqué au problème de la pollution ponctuelle d'origine urbaine. Plusieurs stations de traitement des eaux usées municipales ont été construites dans le cadre de ce programme réduisant de façon importante les charges de polluants d'origine urbaine. La question demeure toutefois de savoir dans quelle mesure les charges urbaines rejetées avant et l'après l'instauration de ce programme peuvent entraîner des dépassements de différents critères de l'eau. La présente étude a pour objectif d'examiner cette problématique pour le bassin versant de la rivière Chaudière en utilisant le système de modélisation intégrée GIBSI (Gestion intégrée par bassin versant à l'aide d'un système informatisé). Deux scénarios d'assainissement urbain ont été examinés, l'un représentatif de la période avant la mise en place du programme, le début des années 1980, et un autre représentatif de la période plus récente. Deux chroniques météorologiques ont été utilisées (années 1983 et 1994). L'estimation des probabilités de dépassement de différents critères de l'eau montre une nette réduction de ces probabilités après mise en place du programme. Pour la $\mathrm{DBO}_{5}$ et l'azote total, les charges urbaines rejetées actuellement n'entraînent pas de dépassement des critères de qualité de l'eau pour les deux années retenues. Toutefois, les résultats montrent que les charges en phosphore total d'origine urbaine peuvent à elles seules entraîner des probabilités de dépassement importantes lors d'étiage.

Mots clés : rejets ponctuels urbains, probabilité de dépassement, QUAL2E, assainissement des eaux.

\section{1 - INTRODUCTION}

Le gouvernement du Québec a mis en place à la fin des années 1970 un ambitieux programme dont l'objectif était la dépollution des rivières du Québec. Ce programme, nommé Programme d'assainissement des eaux du Qué- 
bec (PAEQ), s'est d'abord intéressé à la problématique de la pollution d'origine urbaine. Pour ce faire, des objectifs environnementaux de rejets (OER) ont été définis pour les stations de traitement des eaux usées municipales (SIMONEAU et al., 1998). Ces OER servent à définir les charges et les concentrations qui assureront le respect des critères de qualité de l'eau, et ce afin de permettre la récupération des usages de l'eau et la pérennité et l'intégrité des écosystèmes aquatiques. Ces OER sont généralement établis sur la base de certaines récurrences des débits. ROUSSEAU et al. (2002) présentent une description plus détaillée de la notion de OER. Pour les rejets urbains, du fait du caractère ponctuel et de la relative constance de ces charges en fonction du temps, ces OER se traduisent en exigences de rejets. Ainsi l'ouvrage de traitement des eaux devait permettre une réduction de la charge rejetée au cours d'eau conforme au niveau défini par ces exigences de rejets.

L'impact de ce programme a été majeur quant au nombre de stations construites et à l'augmentation de la population raccordée à un réseau d'égout dont les eaux usées sont traitées. Dans le cas du bassin versant de la rivière Chaudière, il s'agit de plus de $95 \%$ de la population raccordée à un réseau en 1997. La diminution de la charge totale rejetée en rivière a elle aussi été importante. SIMONEAU (1998) rapporte dans son étude avoir constaté une tendance globale à l'amélioration de la qualité de l'eau sur le bassin versant de la rivière Chaudière au cours des deux dernières décennies. Cette amélioration est attribuée pour une bonne part au programme d'assainissement des eaux usées d'origine urbaine et à l'amélioration de l'étanchéité des infrastructures d'entreposage de fumier. De plus, à partir d'un bilan sur les charges d'azote et de phosphore, les apports respectifs des sources diffuses et urbaines ont pu être estimés par cet auteur. II appert que les problèmes résiduels observés sont localisés pour une large part en zone agricole.

D'autres analyses tendent à identifier la pollution diffuse d'origine agricole comme le principal responsable de la mauvaise qualité actuelle des eaux. Mentionnons GANGBAZO et PAINCHAUD (1999) qui, dans une étude sur six bassins versants agricoles du Québec, ont montré que les zones où la pression agricole est importante ne montraient pas de tendance à l'amélioration de la qualité des eaux au cours de la période récente. De même, GANGBAZO (1997) a démontré l'existence d'une relation statistique significative entre occupation du territoire et qualité des eaux, suggérant donc que les pressions agricoles sont les principales sources responsables de la mauvaise qualité observée sur certains bassins.

II semble donc établi parmi la communauté d'experts que la pollution diffuse d'origine agricole est l'élément clef de la problématique actuelle de la qualité des eaux au Québec. Le caractère diffus de ce type de pollution, variable dans le temps et l'espace, rend son analyse scientifique et technique complexe. ROUSSEAU et al. (2002) donnent une description des enjeux liés à cette problématique. Toutefois, même si la mise en place d'un réseau de stations de traitement des eaux usées a conduit à une diminution substantielle des charges d'origine urbaine, il est important de se demander comment ces diminutions se sont traduites en matière de concentrations dans les cours d'eau. Par exemple, est-ce que les seules sources urbaines peuvent conduire à des probabilités de dépassement significatives des critères de qualité de l'eau ? Quel a été l'impact de la mise en place du programme d'assainissement urbain sur les probabilités de dépassement des critères de qualité de l'eau ? Dans 
quelle mesure peut-on affirmer que la pollution d'origine urbaine a une incidence marginale sur la qualité de l'eau en comparaison de celle attribuable à la pollution diffuse d'origine agricole ? II est intéressant de mentionner à ce propos l'étude de MARANDA et SASSEVILLE (1999) qui a examiné une problématique similaire mais en l'abordant d'un point de vue économique et social. Ces auteurs ont effectivement examiné le programme d'assainissement mis en place sur le bassin versant de la rivière Chaudière en se demandant si les investissements effectués ont conduit à une réduction maximale des charges polluantes déversées dans le bassin.

La présente étude a pour principal objectif d'apporter un éclairage sur ces questions à travers une série de simulations visant à évaluer l'impact sur la qualité de l'eau de la réduction des charges ponctuelles d'origine urbaine sur le bassin versant de la rivière Chaudière. Cet impact est évalué en utilisant le système de modélisation intégrée GIBSI (Gestion intégrée par bassin versant à l'aide d'un système informatisé) (ROUSSEAU et al., 2000 ; MAlLHOT et al., 1997 ; VILLENEUVE et al., 1998) qui permet, entre autres, la comparaison de différents scénarios d'assainissement urbain. L'utilisation de GIBSI permet aussi l'évaluation des concentrations journalières en rivière en fonction des conditions variables d'écoulement et en tenant compte des principaux processus hydrobiochimiques affectant ces concentrations. Deux scénarios d'assainissement urbain sont examinés. Le premier correspond à la situation du début des années 1980 où seules quelques stations de traitement des eaux étaient opérationnelles et le deuxième, représentatif de la période récente, où la quasi-totalité des rejets est traitée. La comparaison des impacts de ces différents scénarios se fera en considérant les probabilités de dépassement des critères de l'eau associées aux variables simulées pour deux chroniques météorologiques couvrant une année chacune.

\section{2 - ASSAINISSEMENT DES EAUX USÉES D'ORIGINE URBAINE SUR LE BASSIN VERSANT DE LA RIVIÈRE CHAUDIÈRE}

Le bassin versant de la rivière Chaudière couvre une superficie de $6682 \mathrm{~km}^{2}$ (SIMONEAU et al., 1998). L'occupation du territoire est dominée par la forêt avec $62 \%$ de la superficie, suivie par les terres agricoles qui couvrent $32,6 \%$ du territoire, l'eau 1,7 \% et les zones urbaines 3,6 \% (VILLENEUVE et al., 1997). La population totale du bassin en 1996 était de 173129 habitants. Durant la période 1976-1996, cette population a augmenté de $26 \%$. Des trois secteurs du bassin, celui de la basse Chaudière a connu la plus forte augmentation de population avec $60 \%$.

La figure 1 montre l'évolution des progrès en matière d'assainissement urbain pour le bassin versant de la rivière Chaudière. En 1996, 55 des 77 municipalités du bassin possédaient un réseau d'égout pour une population totale représentant $65 \%$ de celle du bassin. Le pourcentage de la population du bassin raccordée à un réseau d'égout et dont les eaux usées étaient traitées atteignait $95 \%$ en 1997 . On peut constater que le progrès réalisé à ce chapitre est important et a été très rapide. 


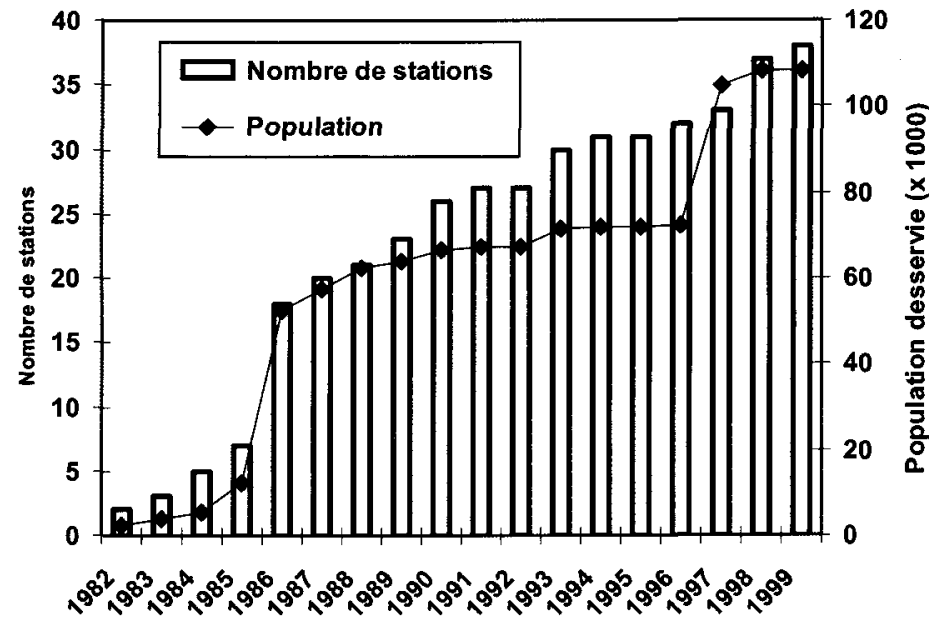

Figure 1 Évolution du nombre de stations de traitement des eaux usées municipales et de la population desservie par ces stations sur le bassin versant de la rivière Chaudière.

Evolution of the number of waste water treatment plants and of the percentage of the connected population for the Chaudière river watershed.

La carte de la figure 2 localise l'ensemble des 44 points de rejets urbains du bassin versant de la rivière Chaudière. La liste de ces derniers est fournie au tableau 1 avec, le cas échéant, le type de station et les traitements associés à ces rejets (Ministère de l'Environnement et de la Faune du Québec, 1997 ; SIMONEAU et al., 1998). Les dates de mise en service et d'avis de conformité sont aussi indiquées. Cette dernière correspond à la date marquant la fin de la période de rodage où divers ajustements ont pu être apportés afin d'obtenir un rendement conforme aux attentes. Un avis est alors émis par le ministère des Affaires municipales qui confirme la fin de la période de rodage. La population indiquée dans ce tableau correspond à la population raccordée au réseau en 1994. Il est à noter que les eaux usées provenant d'industries non raccordées à un réseau municipal ne sont pas considérées dans ce qui suit.

II est intéressant de voir comment les charges d'origine urbaine ont évolué au fil des ans suivant la mise en opération des différentes stations de traitement des eaux usées. Dans ce qui suit, quatre points de contrôle sont considérés, chacun correspondant à la position de l'une des stations principales de mesures de qualité de l'eau sur le bassin (figure 3). Les charges annuelles en phosphore total, en azote total et en $\mathrm{DBO}_{5}$ provenant des rejets urbains situés dans les sous-bassins en amont de chacun de ces points ont été estimées. La figure 4 donne un exemple de cette évolution pour le phosphore total à la station 2340012 (prise d'eau de Charny), station située près de l'exutoire du bassin. L'évolution des charges de $\mathrm{DBO}_{5}$ et d'azote total suit un patron similaire. Les données compilées par le ministère de l'Environnement et de la Faune (MEF) pour les diverses stations de traitement du bassin versant de la Chaudière pour les années 1994 et 1995 ont été utilisées pour l'estimation des charges de phosphore total et de $\mathrm{DBO}_{5}$ (Ministère de l'Environnement et de la 


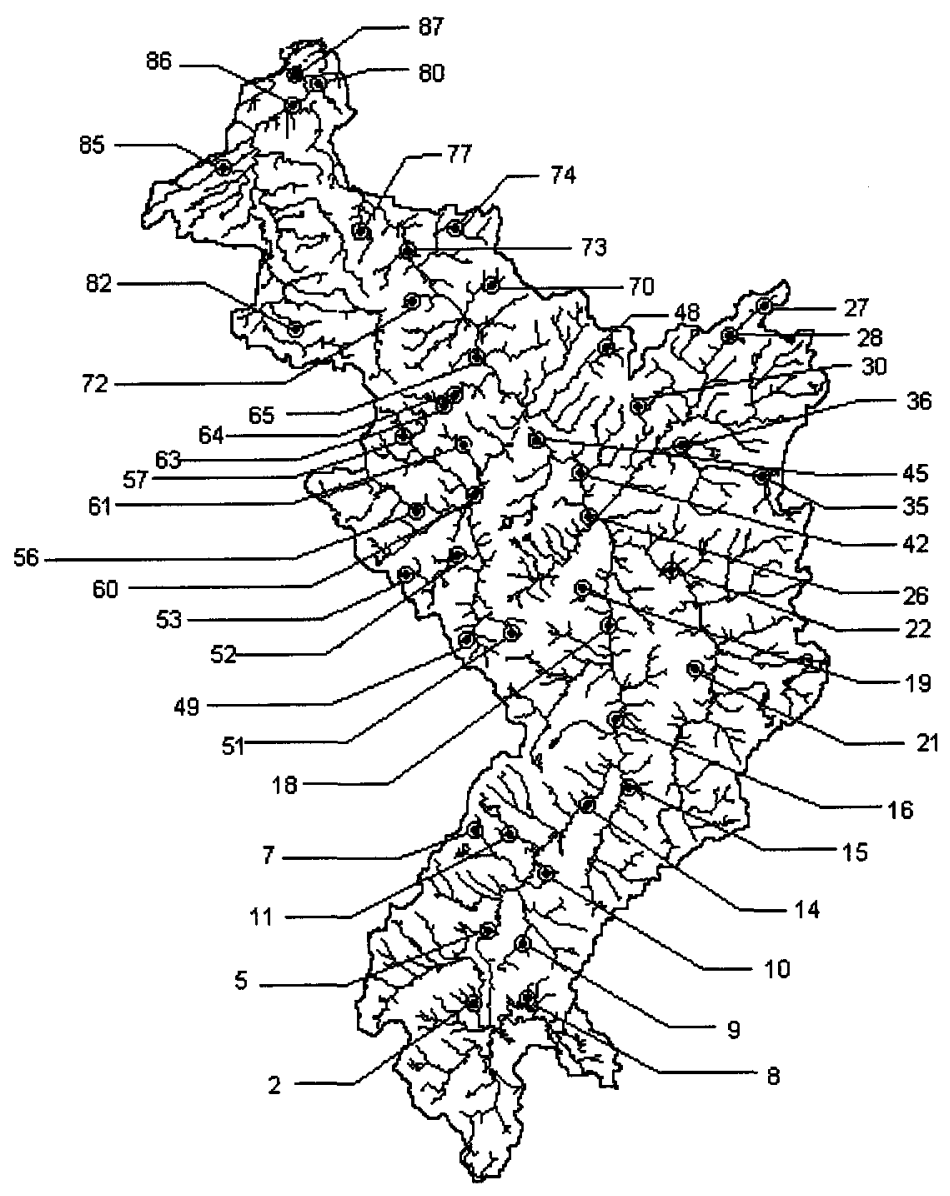

Figure 2 Localisation des points de rejets urbains sur le bassin versant de la rivière Chaudière.

Location of the urban point loads within the Chaudière river watershed.

Faune du Québec, 1997). Ces données établissent un bilan annuel des charges à l'affluent et à l'effluent pour chacune des usines de traitement du bassin. Les valeurs des charges et des rendements diffèrent peu d'une année à l'autre. Les valeurs de 1995 ont donc été utilisées. Les charges à l'affluent en 1995 sont considérées dans les cas où la station de traitement n'était pas en opération et la valeur à l'effluent lorsqu'elle a été mise en service. Les valeurs à l'effluent ont été utilisées aussitôt la mise en opération des stations et aucune période de rodage n'a été considérée. En ce qui concerne les charges d'azote à l'affluent, une concentration de $40 \mathrm{mg}-\mathrm{N} / \mathrm{L}$ d'azote total a été considérée (TCHOBANOGLOUS et SCHOEDER, 1985 ; NOVOTNY et CHESTERS, 1981). Par ailleurs, les données du MEF ont permis d'estimer à $0,73 \mathrm{~m}^{3}$ le volume total moyen des eaux usées produit par jour par personne. Cette valeur inclut les débits domestiques et d'infiltration. Comparée à la valeur moyenne de $320 \mathrm{~L} /($ personne/jour) du débit d'eaux usées domestiques généralement considéré au Québec (BRIĖRE, 
Tableau 1 Liste des rejets urbains présents sur le bassin versant de la rivière Chaudière.

Table $1 \quad$ List of urban point loads within the Chaudière river watershed.

\begin{tabular}{|c|c|c|c|c|c|c|}
\hline $\mathbf{N}^{\circ}$ & Nom & $\begin{array}{c}\text { Population } \\
\text { raccordée } \\
\text { en } 1994\end{array}$ & $\begin{array}{l}\text { Type } \\
\text { de } \\
\text { station }\end{array}$ & $\begin{array}{l}\text { Traite- } \\
\text { ment }^{2}\end{array}$ & $\begin{array}{l}\text { Date de } \\
\text { mise en } \\
\text { service }\end{array}$ & $\begin{array}{c}\text { Date } \\
\text { d'avis de } \\
\text { conformité }\end{array}$ \\
\hline 10 & Audet & 285 & FS & & 82.07 & 89.11 \\
\hline 11 & Lac-Drolet & 735 & EN & & $\begin{array}{l}\text { Avant } \\
\text { PAEQ }\end{array}$ & \\
\hline 14 & $\begin{array}{l}\text { Risborough } \\
\text { (et-partie-de-Marlow) }\end{array}$ & 654 & EA & $P$ & 91.06 & 94.10 \\
\hline 15 & St-Robert-Bellarmin & 353 & EN & & 87.12 & 92.04 \\
\hline 16 & St-Gédéon & 1538 & EA & & 83.10 & 90.02 \\
\hline 18 & St-Martin & 1582 & EA & $P$ & 86.09 & 90.02 \\
\hline 19 & St-Jean-de-la-Lande & 354 & EA & & 96.10 & \\
\hline 2 & Piopolis & 300 & Aucune & & & \\
\hline 21 & St-Théophile & 438 & EA & & 98.03 & \\
\hline 22 & St-Come-Linière & 1978 & EA & $P$ & 86.09 & 91.07 \\
\hline 26 & $\begin{array}{l}\text { St-Georges } \\
\text { (Aubert-Gallion) }\end{array}$ & 17422 & $\mathrm{BA}$ & P/UV & 86.12 & 95.09 \\
\hline 27 & Ste-Justine & 1622 & EA & $P$ & 93.11 & 96.03 \\
\hline 28 & $\begin{array}{l}\text { Ste-Germaine- } \\
\text { du-Lac-Etchemin }\end{array}$ & 340 & EA & $P$ & 93.12 & 96.04 \\
\hline 30 & St-Benjamin & 708 & Aucune & & & \\
\hline 35 & Ste-Aurélie & 2185 & EA & & 82.10 & 89.12 \\
\hline 36 & St-Prosper & 2463 & EA & $P$ & 86.12 & 91.07 \\
\hline 42 & Notre-Dame-des-Pins & 513 & $\mathrm{BD}$ & P/UV & 86.09 & 92.12 \\
\hline 45 & Beauceville & 3785 & BA & P/UV & 86.11 & 93.09 \\
\hline 48 & $\begin{array}{l}\text { St-Odilon-de- } \\
\text { Cranbourne }\end{array}$ & 976 & EA & $P$ & 89.09 & 92.03 \\
\hline 49 & La Guadeloupe & 1566 & EA & $P$ & 86.12 & 95.02 \\
\hline 5 & Lac Mégantic & 6195 & BA & $P$ & 85.07 & 95.09 \\
\hline 51 & Shenley & 1009 & EA & $P$ & 86.12 & 95.03 \\
\hline 52 & $\begin{array}{l}\text { St-Ephrem-de- } \\
\text { Beauce }\end{array}$ & 1062 & EA & $P$ & 86.11 & 91.07 \\
\hline 53 & $\begin{array}{l}\text { St-Méthode-de- } \\
\text { Frontenac }\end{array}$ & 970 & EA & & 84.12 & 94.06 \\
\hline 56 & $\begin{array}{l}\text { Ste-Chlothilde-de- } \\
\text { Beauce }\end{array}$ & 247 & Aucune & & & \\
\hline 57 & East-Broughton & 1798 & EA & $P$ & 90.12 & 93.06 \\
\hline 60 & St-Victor & 1352 & EA & & 98.09 & \\
\hline 61 & St-Jules & 153 & Aucune & & & \\
\hline 63 & Tring-Jonction & 1278 & EA & $P$ & 86.09 & 93.08 \\
\hline 64 & St-Frédéric & 564 & Aucune & & & \\
\hline 65 & $\begin{array}{l}\text { St-Joseph-de- } \\
\text { Beauce }\end{array}$ & 4986 & EA & $P$ & 88.08 & 93.05 \\
\hline 7 & $\begin{array}{l}\text { Ste-Cécile-de- } \\
\text { Whitton St-Samuel }\end{array}$ & 356 & Aucune & & & \\
\hline
\end{tabular}


Tableau 1 (suite) Liste des rejets urbains présents sur le bassin versant de la rivière Chaudière.

Table 1 (continued) List of urban point loads within the Chaudière river watershed.

\begin{tabular}{|c|c|c|c|c|c|c|}
\hline $\mathbf{N}^{\circ}$ & Nom & $\begin{array}{c}\text { Population } \\
\text { raccordée } \\
\text { en } 1994\end{array}$ & $\begin{array}{c}\text { Type } \\
\text { de } \\
\text { station'1 }\end{array}$ & $\begin{array}{l}\text { Traite- } \\
\text { ment }^{2}\end{array}$ & $\begin{array}{l}\text { Date de } \\
\text { mise en } \\
\text { service }\end{array}$ & $\begin{array}{c}\text { Date } \\
\text { d'avis de } \\
\text { conformité }\end{array}$ \\
\hline 70 & Saints-Anges & 377 & EA & $P$ & 94.09 & 97.11 \\
\hline 72 & St-Elzéar & 897 & EA & $P$ & 90.12 & 92.10 \\
\hline 73 & Ste-Marie & 7664 & EA & $P$ & 86.03 & 96.12 \\
\hline 74 & Ste-Marguerite & 399 & EN & & 89.06 & 91.09 \\
\hline 77 & St-Bernard & 714 & $B A$ & $P$ & 85.05 & 88.10 \\
\hline 8 & Frontenac (Mercier) & 125 & EN & $P$ & 90.09 & 94.06 \\
\hline 80 & $\begin{array}{l}\text { Ste-Hélène-de } \\
\text { Breakeyville }\end{array}$ & 2740 & EA & & $\begin{array}{l}\text { Avant } \\
\text { PAEQ }\end{array}$ & \\
\hline 82 & St-Sylvestre & 327 & EA & & 98.09 & \\
\hline 85 & St-Agapit & 2262 & EA & $P$ & 93.11 & 96.12 \\
\hline 86 & $\begin{array}{l}\text { St-Étienne-de- } \\
\text { Lauzon }\end{array}$ & 4463 & EA & $P$ & 87.12 & 91.12 \\
\hline 87 & Charny & 32613 & $B A$ & UV & 97.11 & \\
\hline 9 & $\begin{array}{l}\text { Frontenac village } \\
\text { (rang 3) }\end{array}$ & 305 & EN & 84.10 & 90.09 & \\
\hline
\end{tabular}

1. EA : étangs aérés ; $E N$ : étangs non aérés ; $B A$ : boues activées ; FS : fosse septique.

2. $P$ : déphosphotation ; UV : ultraviolet.

1997), une telle valeur est sans doute symptomatique d'infiltration importante sur ces réseaux. Les charges en azote total à l'affluent ont donc été calculées à partir de ces données de base. Il est à noter toutefois que, compte tenu des infiltrations significatives en réseau, la concentration considérée de $40 \mathrm{mg}-\mathrm{N} / \mathrm{L}$, qui correspond à la concentration moyenne domestique, surestime sans doute la concentration effective en azote des eaux à leur arrivée à l'usine. Cette valeur a toutefois été utilisée faute de données plus précises sur ces concentrations. Quant aux rendements des différents types de station pour le prélèvement des composés azotés, ils proviennent de données compilées par le MEF. Le tableau 2 présente un résumé de ces rendements. Par ailleurs, aucune donnée n'est disponible concernant l'évolution des populations raccordées au réseau. Pour estimer l'évolution de cette population, nous avons simplement considéré la croissance démographique du bassin pendant la période à l'étude et supposé que la fraction de population raccordée au fil des années demeurait inchangée.

La forme générale des histogrammes des charges annuelles totales aux autres points de contrôle est sensiblement la même que celle de la figure 4 . Dans tous les cas, la période autour de l'année 1986 marque un tournant où la diminution des charges est la plus importante. De part et d'autre de cette période, les charges varient beaucoup plus lentement. Deux années de référence ont donc été retenues : 1983 et 1994. Le choix de ces deux années se justifie par le fait qu'elles sont pour l'une représentative de la situation préva- 


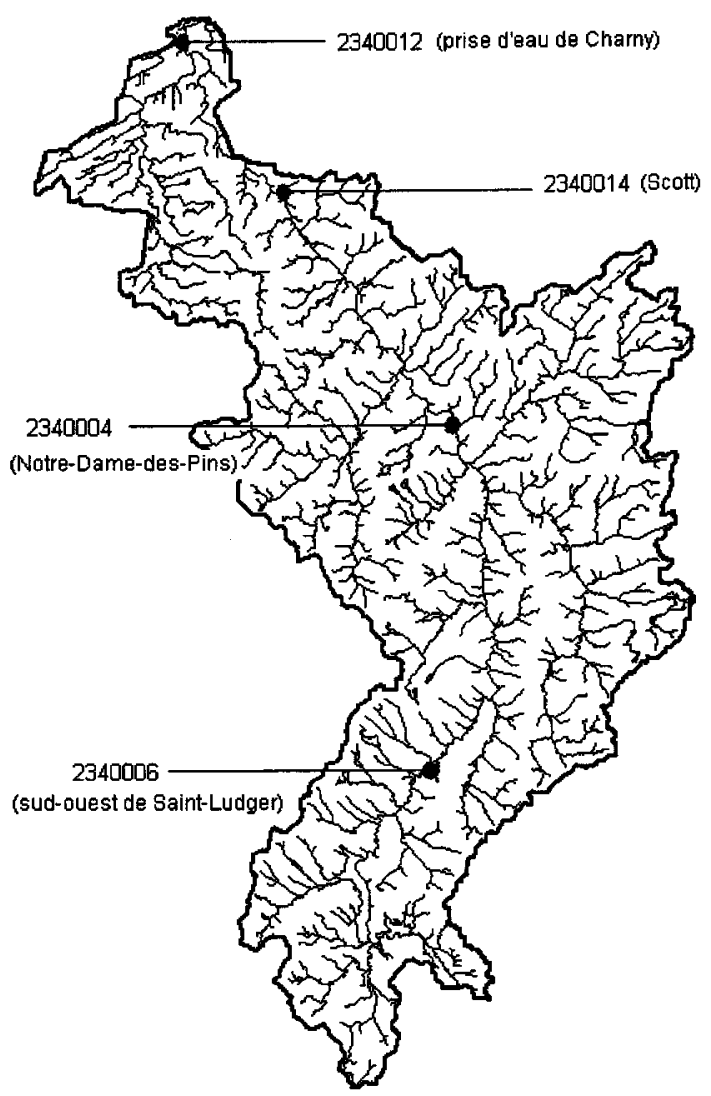

Figure 3 Localisation des quatre points de contrôle correspondant aux stations principales de mesures de la qualité de l'eau sur le bassin versant de la rivière Chaudière.

Location of the main water quality monitoring stations within the Chaudière river watershed.

Tableau 2 Rendement des différents types de stations pour le traitement des charges d'azote.

Table 2 Nitrogen removal efficiency for different types of waste water treatment plants.

\begin{tabular}{|c|c|c|}
\hline $\begin{array}{c}\text { Type de } \\
\text { traitement }\end{array}$ & $\begin{array}{c}\text { Rendement } \\
\text { d'enlèvement }\end{array}$ & $\begin{array}{c}\text { Forme et pourcentage à l'effluent } \\
\text { de la fraction non prélevée }\end{array}$ \\
\hline EA et EN & $50 \%$ & $\begin{array}{c}44 \% \text { en } \mathrm{NH}_{4} \\
6 \% \text { en NO}\end{array}$ \\
\hline BA & $53 \%$ & $21 \%$ en $\mathrm{NH}_{4}$ \\
& & $32 \%$ en $\mathrm{NO}_{\mathrm{x}}$ \\
\hline
\end{tabular}

1. EA : étangs aérés ; EN : étangs non aérés ; $B A$ : boues activées

2. Le rendement d'enlèvement se définit comme suit : (1 - charge d'effluent/charge d'affluent). 


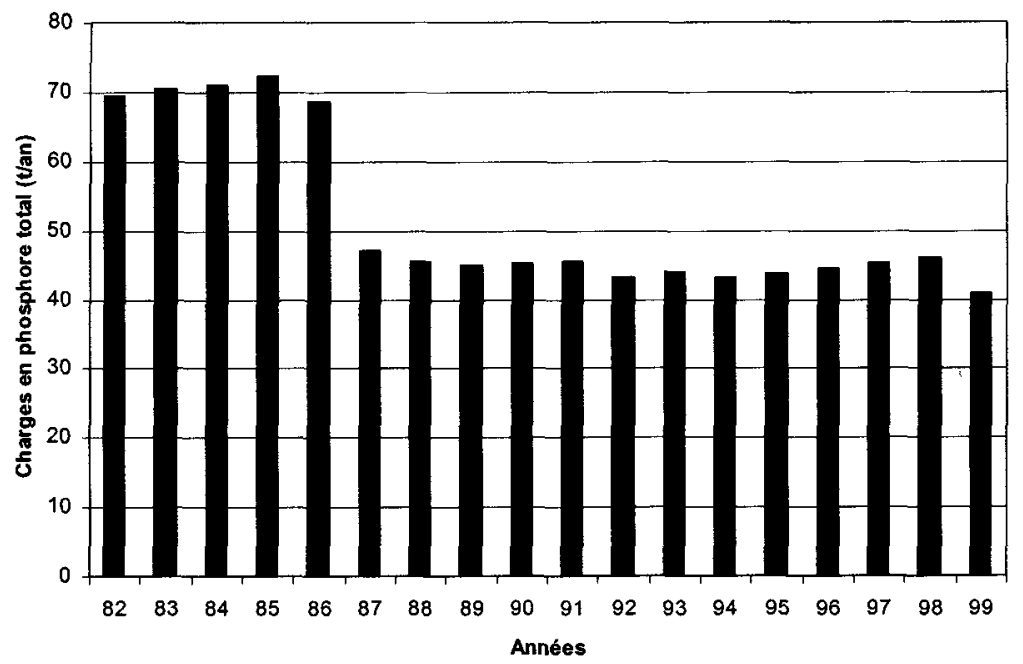

Figure 4 Évolution des charges annuelles en phosphore total rejetées au cours d'eau pour le sous-bassin versant délimité par la station 2340012 (prise d'eau de Charny).

Annual discharged loads of total phosphorus for the sub-watershed of station 2340012 (Charny's water uptake).

Tableau 3 Charges annuelles d'origine urbaine rejetées dans les différents sousbassins délimités par les points de contrôle pour les années 1983 et 1994 (toutes les charges sont exprimées en tonnes/an).

Table 3 Annual urban loads discharged within the different sub-watersheds of the control points for years 1983 and 1994 (in tons/year).

\begin{tabular}{|c|c|c|c|}
\hline \multirow{2}{*}{ Station } & \multirow{2}{*}{ Variables } & \multicolumn{2}{|c|}{ Année } \\
\hline & & 1983 & 1994 \\
\hline 2340006 & $\begin{array}{c}\text { Phosphore total } \\
\text { Azote total } \\
\mathrm{DBO}_{5}\end{array}$ & $\begin{array}{c}6,2 \\
60 \\
247,5\end{array}$ & $\begin{array}{c}2,6 \\
36,5 \\
43\end{array}$ \\
\hline 2340004 & $\begin{array}{l}\text { Phosphore total } \\
\text { Azote total } \\
\mathrm{DBO}_{5}\end{array}$ & $\begin{array}{c}30,6 \\
321,7 \\
974,8\end{array}$ & $\begin{array}{c}13,1 \\
202,7 \\
201,7\end{array}$ \\
\hline 2340014 & $\begin{array}{l}\text { Phosphore total } \\
\text { Azote total } \\
\mathrm{DBO}_{5}\end{array}$ & $\begin{array}{c}45,5 \\
514,5 \\
2048,6\end{array}$ & $\begin{array}{r}20,9 \\
323,8 \\
344,6\end{array}$ \\
\hline 2340012 & $\begin{array}{l}\text { Phosphore total } \\
\text { Azote total } \\
\mathrm{DBO}_{5}\end{array}$ & $\begin{array}{c}70,4 \\
822,5 \\
3056,8\end{array}$ & $\begin{array}{c}43,3 \\
520 \\
511,7\end{array}$ \\
\hline
\end{tabular}

lant avant la mise en place du programme d'assainissement et pour l'autre de la situation après la mise en place de ce programme. Le tableau 3 présente les charges totales d'origine urbaine rejetées aux cours d'eau pour ces deux 
années dans les bassins versants délimités par les quatre points de contrôle. Pour la station la plus en aval (station 2340012 ; prise d'eau de Charny), on constate une réduction des charges sur cette période de l'ordre de $38 \%$ pour le phosphore total, de $37 \%$ pour l'azote total et de $83 \%$ pour la $\mathrm{DBO}_{5}$.

\section{3 - SYSTÈME DE MODÉLISATION INTÉGRÉE GIBSI}

Le système GIBSI est un outil de modélisation dont l'objectif premier est l'évaluation des impacts de différents types d'aménagement ou différentes options de gestion de l'eau et du territoire sur la qualité de l'eau à l'échelle du bassin versant (ROUSSEAU et al. 2000 ; VILLENEUVE et al., 1998 ; MAILHOT et al., 1997). GIBSI intègre un ensemble de modèles capable de simuler les processus hydrologiques (HYDROTEL : FORTIN et al., 1995 ; FORTIN et al. 2001a), d'érosion hydrique des sols (version révisée de USLE : WISCHMEIR et SMITH, 1978 ; RENARD et al., 1997), de transport des polluants agricoles (adaptation des modèles SWAT et EPIC : ARNOLD et WILLIAMS, 1995 ; WILLIAMS, 1995) et de qualité de l'eau (QUAL2E : BROWN et BARNWELL, 1987). Chacun de ces modèles a été modifié et adapté aux besoins du présent système tant pour uniformiser la représentation spatiale du réseau que pour permettre un couplage entre ces modèles (VILLENEUVE et al., 1998). À titre d'exemple, le modèle QUAL2E a été révisé afin de pouvoir simuler des conditions d'écoulement variables dans le temps. Le pas de temps utilisé pour l'ensemble des modèles est la journée.

Le système permet de considérer quatre grandes classes de scénarios d'aménagement, à savoir des scénarios :

- d'aménagement du territoire ;

- de gestion de la pollution diffuse agricole ;

- de gestion des rejets ponctuels urbains et industriels ;

- de gestion des barrages.

L'usager peut en effet modifier les paramètres liés à l'un ou l'autre de ces scénarios (ROUSSEAU et al., 2000). Dans le cas des rejets urbains, l'usager peut modifier la population raccordée, les types de traitement, et peut aussi considérer la situation où les eaux usées municipales ne sont pas traitées. II est aussi possible de spécifier les débits et concentrations en un ou plusieurs points de rejets pendant quelques jours afin de simuler, par exemple, un débordement de réseau d'égout ou une mise hors service d'une station de traitement.

Le calage du modèle hydrologique est décrit dans FORTIN et al. (2001b). Globalement, des valeurs du coefficient de Nash-Sutcliffe comprises entre 0,71 et 0,89 ont été obtenues tant lors du calage que de la validation. De plus, les volumes annuels moyens simulés par le modèle sont à moins de $15 \%$ des valeurs estimées à partir des mesures (FORTIN et al., 2001b).

Le calage du modèle de qualité de l'eau est un exercice plus difficile compte tenu des importantes incertitudes sur les données d'entrée du modèle 
particulièrement en ce qui concerne les apports diffus et du peu de données disponibles. Des valeurs de paramètres du modèle de qualité ont donc été choisies à l'intérieur des intervalles de valeurs suggérés par la littérature (BROWN et BARNWELL, 1987 ; BOWIE et al. 1985) et un travail de validation de l'approche a donc été réalisé afin de vérifier que les résultats obtenus à l'aide du modèle se comparaient globalement aux valeurs mesurées en rivière. Cet exercice a permis de valider le choix des paramètres et les hypothèses retenues relativement à certaines données sur les apports diffus pour lesquels aucune donnée n'est disponible (e.g. dates et volumes des épandages, modes d'épandage). Cette méthodologie de validation du système GIBSI est décrite dans TURCOTTE et al. (1999), ROUSSEAU et al. (2000) et ROUSSEAU et al. (2002).

\section{4 - SCÉNARIOS ET CHRONIQUES MÉTÉOROLOGIQUES CONSIDÉRÉS}

Afin d'évaluer l'impact de la diminution des charges urbaines sur les concentrations en rivière, deux scénarios ont été considérés. Un premier se veut représentatif de la période récente. Ce scénario reprend la situation de l'année 1994 et considère opérationnelles uniquement les stations en service en 1994. L'autre scénario cherche à décrire la situation antérieure à la mise en place du programme d'assainissement et considère opérationnelles les stations mises en service avant 1983. Seules quatre stations de traitement étaient alors en service, soient celles d'Audet, de Ste-Aurélie, du Lac Drolet et de Sainte-Hélène-de-Breakeyville (\# 10, \# 35, \#11 et \# 80 sur la figure 1). Il est important de noter qu'aucun apport diffus d'origine agricole ou urbaine n'est considéré dans ce qui suit, pas plus que les apports d'origine naturelle.

Tableau 4 Volumes d'eau annuels et estivaux ( 21 juin au 20 septembre) ayant transités par les points de contrôle pour les années 1983 et 1994.

Table 4 Annual and summer (June 21st to September 20th) water volumes passing through the control points for years 1983 and 1994.

\begin{tabular}{|c|c|c|c|c|}
\hline \multirow{2}{*}{ Station } & \multicolumn{3}{|c|}{ Volume d'eau $\left(\mathbf{1 0}^{\mathbf{6}} \mathbf{~ m}^{\mathbf{3}}\right)$} \\
\hline & \multicolumn{2}{|c|}{$\mathbf{1 9 8 3}$} & \multicolumn{2}{c|}{$\mathbf{1 9 9 4}$} \\
\hline & Annuel & Période estivale & Annuel & Période estivale \\
\hline 2340006 & 555,7 & 25,2 & 641,6 & 113,8 \\
2340004 & 1660,5 & 66,1 & 2291,9 & 381 \\
2340014 & 2515,1 & 108,2 & 3583,1 & 729,3 \\
2340012 & 2967,8 & 131,2 & 4299,3 & 901,1 \\
\hline
\end{tabular}

Le tableau 5 présente la nomenclature des diverses simulations effectuées en fonction des scénarios et chroniques météorologiques retenus. L'analyse qui suit ne considère que les quatre points de contrôle correspondant aux 

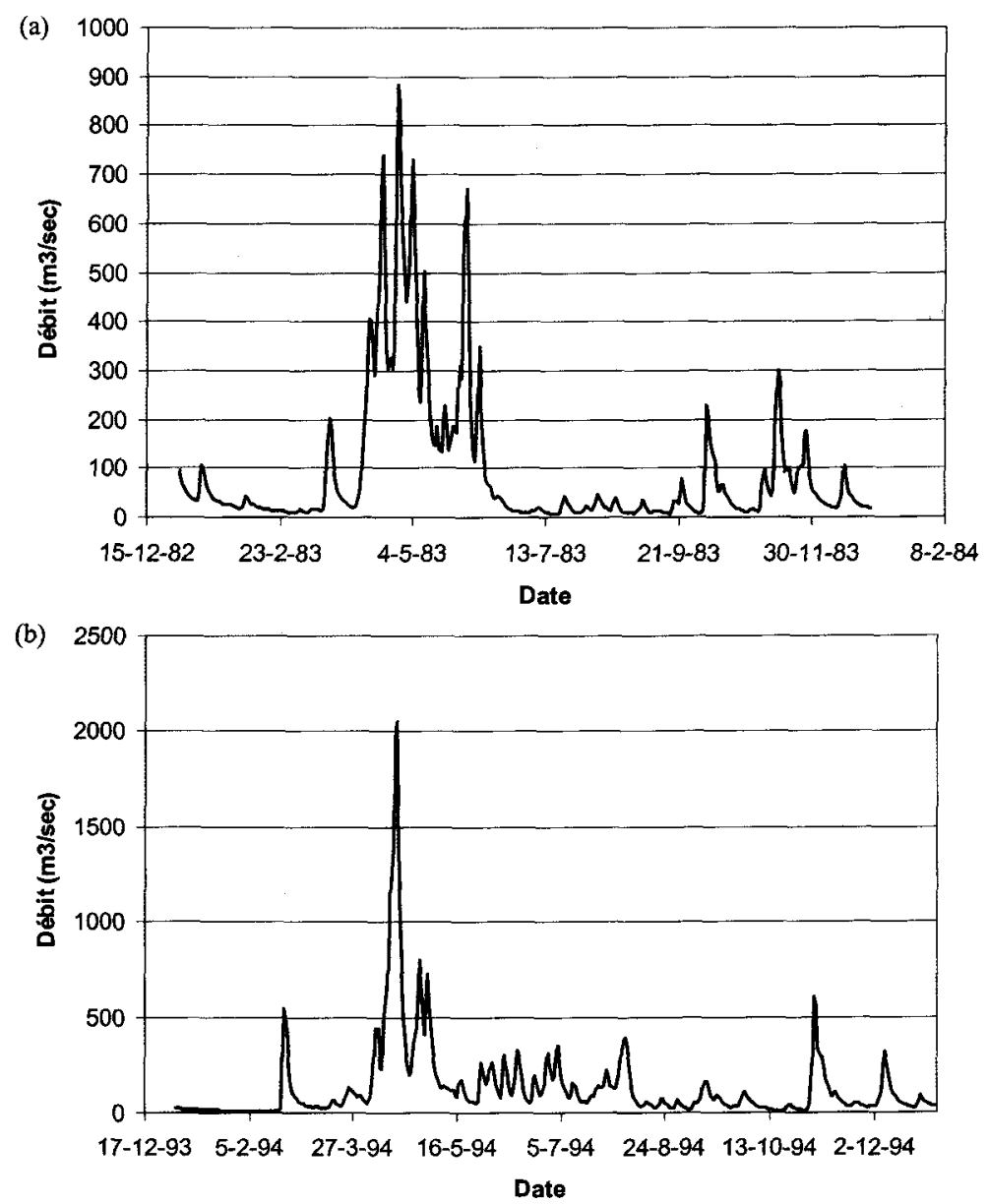

Figure 5 Débits simulés à la station 2340012 (prise d'eau de Charny) pour les années (a) 1983 et (b) 1994.

Simulated flows at station 2340012 (Charny's water uptake) for years (a) 1983 and (b) 1994.

positions des stations principales de qualité du bassin pour fin de comparaison entre les diverses simulations (figure 3).

Tableau 5 Nomenclature des simulations effectuées.

Table $5 \quad$ Nomenclature of the simulations.

\begin{tabular}{|c|c|c|}
\hline \multirow{2}{*}{ Scénario } & \multicolumn{2}{|c|}{ Chronique météorologique } \\
\cline { 2 - 3 } & Année 1983 & Année 1994 \\
Rejets 83 & R83A83 & R83A94 \\
Rejets 94 & R94A83 & R94A94 \\
\hline
\end{tabular}




\section{5 - PRÉSENTATION ET ANALYSE DES RÉSULTATS}

La figure 6 présente un exemple de résultat. Ce graphique montre l'évolution de la concentration de phosphore total à la station 2340012 (prise d'eau de Charny) pour les simulations R94A94 et R83A94 (voir tableau 5). Comme on peut le constater, l'impact de la réduction des charges d'origine urbaine est important. La comparaison avec la figure 5 des débits simulés en 1994 montre que les concentrations résultantes sont essentiellement déterminées par le débit, ou en d'autres mots, par le pouvoir de dilution du cours d'eau.

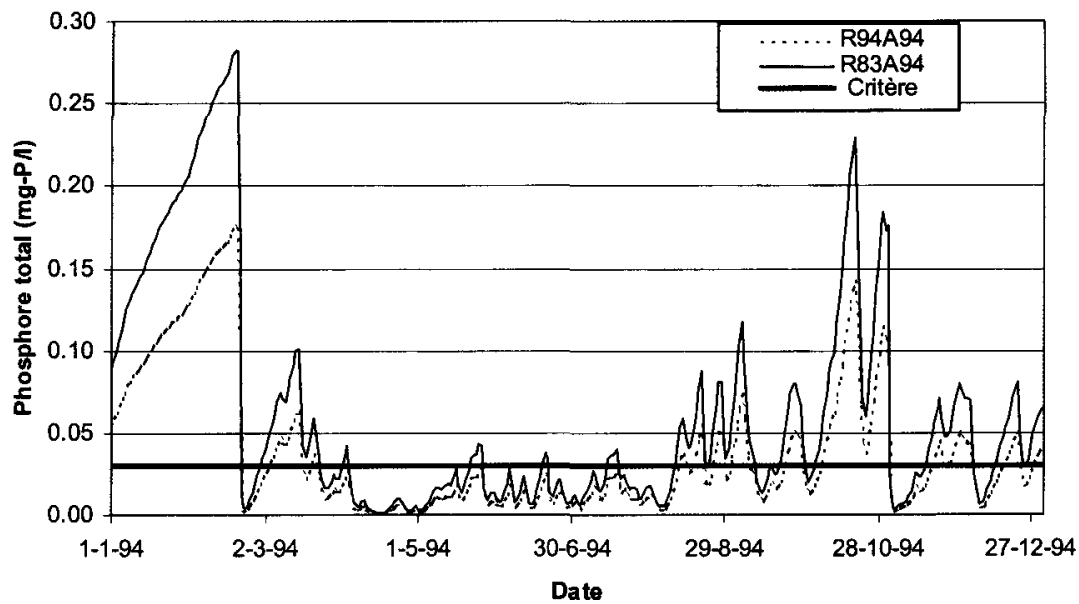

Figure 6 Résultat de simulation : concentration de phosphore total à la station 2340012 (prise d'eau de Charny).

Simulation results: total phosphorus concentration at station 2340012 (Charny's water uptake).

\subsection{Bilan des charges aux différents points de contrôle}

Le premier élément à considérer est le bilan des charges en chacun des points de contrôle. Le tableau 6 présente ces bilans annuels pour chacun des points de contrôle et chacune des simulations. Dans le contexte de simulation de QUAL2E, trois facteurs ont une incidence sur le bilan global du phosphore et de l'azote :

- les apports benthiques ;

- la déposition ;

- et les échanges avec les algues (croissance et respiration) (BROWN et BARNWELL, 1987 ; BOWIE et al. 1985).

Compte tenu du peu de données dont nous disposons sur les sources benthiques, nous avons fixé à zéro ce type d'apport. Par ailleurs, pour la déposition, les valeurs minimales des fourchettes proposées par BROWN et BARNWELL (1987) ont été retenues pour les paramètres décrivant ces échanges. 
La comparaison des tableaux 3 et 6 montre que les charges annuelles de phosphore et d'azote total qui transitent par les points de contrôle sont inférieures aux charges totales rejetées dans les sous- bassins en amont de chacun de ces points. Une partie de l'azote et du phosphore se retrouve donc sous une autre forme ou n'est pas acheminée en rivière. De fait, une partie de ces composés est assimilée par les algues et le modèle QUAL2E considère la déposition des algues. Ce processus dépend localement de la profondeur d'écoulement et de la concentration des algues. Cette déposition fait en sorte qu'une partie du phosphore et de l'azote rejetée au cours d'eau n'est pas acheminée en rivière en aval des points de rejet. Le bilan massique effectué aux points de contrôle montre qu'effectivement la masse totale de ces composés transitant à ces points est inférieure à la masse totale déversée au cours d'eau. On note par ailleurs que l'année 1983, qui est plus sèche, favorise un bilan déficitaire plus important puisque les temps de séjour sont plus grands en moyenne pour cette année.

Tableau 6 Charges annuelles en chacun des points de contrôle pour les différentes simulations.

Table 6 Annual loads at the control points for the different simulations.

\begin{tabular}{|c|c|c|c|c|c|}
\hline \multirow{2}{*}{ Station } & \multirow{2}{*}{ Variables } & \multicolumn{4}{|c|}{ Simulation } \\
\hline & & R83A83 & R83A94 & R94A83 & R94A94 \\
\hline \multirow{3}{*}{2340006} & Phosphore total & 5,5 & 5,8 & 2,2 & 2,6 \\
\hline & Azote total & 54,6 & 68,7 & 38 & 48,2 \\
\hline & $\mathrm{DBO}_{5}$ & 132,5 & 139,9 & 20,6 & 21,7 \\
\hline \multirow{3}{*}{2340004} & Phosphore total & 27,3 & 27,4 & 12 & 12,6 \\
\hline & Azote total & 282 & 291,7 & 187,6 & 195,6 \\
\hline & $\mathrm{DBO}_{5}$ & 539,8 & 571,1 & 107,7 & 11,2 \\
\hline \multirow{3}{*}{2340014} & Phosphore total & 42,8 & 42,8 & 19 & 20 \\
\hline & Azote total & 490,6 & 498,3 & 314 & 320,8 \\
\hline & $\mathrm{DBO}_{5}$ & 1082,5 & 1164,4 & 149 & 167 \\
\hline \multirow{3}{*}{2340012} & Phosphore total & 67,3 & 67,4 & 40,8 & 42,2 \\
\hline & Azote total & 796,6 & 806 & 506,9 & 514,8 \\
\hline & $\mathrm{DBO}_{5}$ & 1594,4 & 1704,6 & 252 & 270,1 \\
\hline
\end{tabular}

Le modèle QUAL2E s'appuie sur la description du bilan de masse pour un volume d'eau en rivière. Les échanges, comme nous le disions précédemment, avec le fond des cours d'eau sont simplement décrits par une série de paramètres et le modèle n'effectue pas de bilan à ce chapitre (BOWIE et al., 1985). De plus, la représentation de ces échanges est statique. D'autres modèles, par exemple WQRRS (SMITH, 1978) et CE-QUAL-R1 (WES, 1982), considèrent un tel bilan et, de ce fait, représentent les sédiments comme un « réservoir dynamique " qui alimente le cours d'eau. Dans un contexte de simulation sur de longues périodes, une telle représentation serait sans doute plus appropriée et permettrait d'assurer un bilan des échanges avec les sédiments plus réaliste. 
Dans le cas de la $\mathrm{DBO}_{5}$, la situation est similaire. Le modèle QUAL2E utilise une cinétique du premier ordre pour décrire l'évolution de cette variable (BROWN et BARNWELL, 1987). Deux types de processus sont pris en compte dans l'estimation des concentrations de $\mathrm{DBO}_{5}$ :

1. la déoxygénation ;

2. le bilan des échanges avec les sédiments (sédimentation, remise en suspension, floculation).

Le bilan dépend donc des valeurs de ces deux paramètres (BROWN et BARNWELL, 1987). Dans un tel contexte, la charge résiduelle en chaque point de contrôle sera déterminée par le temps moyen de séjour dans chacun des sousbassins. Ce temps sera globalement plus court si les débits, et donc les volumes d'eau ayant transités en un point pendant une période donnée, sont plus importants, ce que montre le tableau 6. L'année 1983 est caractérisée par un volume annuel plus petit que l'année 1994 et de fait la charge résiduelle de $\mathrm{DBO}_{5}$ est plus petite aux divers points de contrôle.

\subsection{Probabilité de dépassement aux points de contrôle}

La comparaison des divers scénarios et résultats de simulation s'est effectuée en considérant les probabilités de dépassement (ou le complément de la fonction cumulative) définies par :

$$
F(x)=P(x>x)=1-\int_{0}^{x} d X f(X)
$$

$P(X>x)$ est donc la probabilité que la variable $X$ soit supérieure à $x$ et $f(X)$ est la densité de probabilité de la variable $X$. Le calcul de cette probabilité à partir des données de concentrations simulées sur une période $T$ s'effectue simplement en faisant le rapport entre le nombre de jours où la concentration simulée est supérieure à une concentration de référence et le nombre total de jours de la période T. La figure 7 montre un graphique où les probabilités de dépassement pour le phosphore total à la station 2340012 (prise d'eau de Charny) sont comparées. Les deux courbes correspondent aux simulations R83A94 et R94A94, c'est-à-dire, en matière de rejets ponctuels, aux situations des années 1983 et 1994 respectivement.

Deux critères de qualité de l'eau ont été retenus pour fin de comparaison, un pour le phosphore total et un autre pour la $\mathrm{DBO}_{5}$ (Ministère de l'Environnement et de la Faune, 1992). Le tableau 7 présente un résumé de ces critères de qualité et décrit les usages auxquels ils font références. La figure 7 présente, à titre indicatif, le critère de qualité pour le phosphore total.

Les tableaux 8 et 9 présentent un résumé des résultats obtenus en considérant, pour le premier, une base annuelle pour le calcul des probabilités de dépassement et, pour le second, la période estivale seulement. Cette distinction entre la période annuelle et estivale est utile lorsque l'on se souvient que la période estivale est l'occasion au Québec d'un nombre d'usages récréatifs de l'eau beaucoup plus important et diversifié que pendant les autres périodes de l'année. Les valeurs répertoriées correspondent aux probabilités de dépassement des critères de qualité de l'eau. 


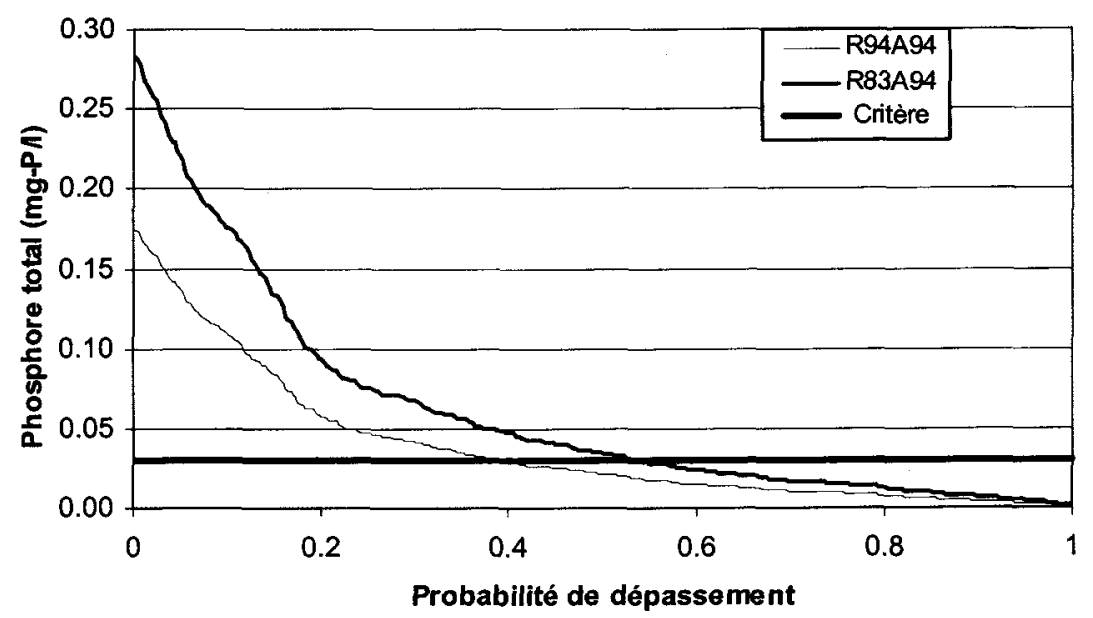

Figure 7 Probabilité de dépassement pour le phosphore total à la station 2340012 (prise d'eau de Charny) pour l'année 1994 considérant les situations 83 et 94 pour les rejets ponctuels (simulations R94A94 et R83A94).

Probability of exceeding WQS for total phosphorus at station 2340012 (Charny's water uptake) for year 1994 under 1983 and 1994 point load conditions (simulations R94A94 and R83A94).

Tableau 7 Critères de qualité de l'eau retenus pour fin de comparaison (d'après Ministère de l'Environnement et de la Faune, 1992).

Table 7 Water quality standards used to analyze simulation results (from Ministère de l'Environnement et de la Faune, 1992).

\begin{tabular}{|c|c|c|}
\hline Variable & Critère & Usage \\
\hline Phosphore total & $0,03 m g-P / L$ & $\begin{array}{l}\text { Vie aquatique, toxicité chronique : } \\
\text { éviter la croissance de plantes } \\
\text { aquatiques dans les rivières }\end{array}$ \\
\hline $\mathrm{DBO}_{5}$ & $3,0 \mathrm{mg} / \mathrm{L}$ & $\begin{array}{l}\text { Eau brute pour application d'un } \\
\text { simple traitement de désinfection }\end{array}$ \\
\hline
\end{tabular}

Pour le phosphore total, à l'échelle annuelle, la probabilité de dépassement du critère à la station 2340012 (prise d'eau de Charny) est de 0,53 pour la simulation R83A94 et de 0,395 pour la simulation R94A94 (figure 7). On constate donc une réduction du nombre de jours avec dépassement du critère à la station 2340012 (prise d'eau de Charny) de l'ordre de 49 jours pour l'année 1994. Des réductions similaires sont observées aux autres points de contrôle. Pour la période estivale, la réduction est beaucoup moins importante avec une probabilité de dépassement de 0,24 pour la simulation R83A94 et de 0,21 pour la simulation R94A94 à la station 2340012 (prise d'eau de Charny). Ces résultats montrent que les sources urbaines de phosphore total peuvent à elles seules entraîner des probabilités de dépassement importantes même dans le contexte actuel de l'assainissement urbain. Pour la chronique météorologique 
Tableau 8 Probabilités de dépassement sur une base annuelle des critères de qualité de l'eau (tableau 5) pour le phosphore total et la $\mathrm{DBO}_{5}$ aux différents points de contrôle.

Table 8 Annual probabilities of exceeding water quality standards (table 5) for total phosphorus and $B O D_{5}$ at the different control points.

\begin{tabular}{|c|c|c|c|c|c|}
\hline \multirow{2}{*}{ Station } & \multirow{2}{*}{ Variables } & & \multicolumn{2}{|c|}{ Simulation } \\
\cline { 3 - 6 } & & $\mathbf{R 8 3 A 8 3}$ & $\mathbf{R 8 3 A 9 4}$ & $\mathbf{R 9 4 A 8 3}$ & $\mathbf{R 9 4 A 9 4}$ \\
\hline \multirow{2}{*}{2340006} & Phosphore total & 0,55 & 0,42 & 0,19 & 0,12 \\
& DBO $_{5}$ & 0 & 0 & 0 & 0 \\
& Phosphore total $_{3} 340004$ & 0,69 & 0,53 & 0,44 & 0,26 \\
& DBO $_{5}$ & 0,03 & 0,06 & 0 & 0 \\
& Phosphore total $_{3}^{2340014}$ & 0,66 & 0,48 & 0,42 & 0,23 \\
& DBO $_{5}$ & 0,08 & 0,13 & 0 & 0 \\
& Phosphore total $^{3}$ & 0,715 & 0,53 & 0,62 & 0,395 \\
& DBO $_{5}$ & 0,22 & 0,16 & 0 & 0 \\
\hline
\end{tabular}

Tableau 9 Probabilités de dépassement pour la période estivale des critères de qualité de l'eau (tableau 5) pour le phosphore total et la $\mathrm{DBO}_{5}$ aux différents points de contrôle.

Table 9 Summer probabilities of exceeding water quality standards (table 5) for total phosphorus and $B O D_{5}$ at the different control points.

\begin{tabular}{|c|c|c|c|c|c|}
\hline \multirow{2}{*}{ Station } & \multirow{2}{*}{ Variables } & \multicolumn{4}{|c|}{ Simulation } \\
\hline & & R83A83 & R83A94 & R94A83 & R94A94 \\
\hline 2340006 & $\begin{array}{l}\text { Phosphore total } \\
\qquad \mathrm{DBO}_{5}\end{array}$ & $\begin{array}{c}0,9 \\
0\end{array}$ & $\begin{array}{c}0,24 \\
0\end{array}$ & $\begin{array}{c}0,33 \\
0\end{array}$ & $\begin{array}{l}0 \\
0\end{array}$ \\
\hline 2340004 & $\begin{array}{l}\text { Phosphore total } \\
\mathrm{DBO}_{5}\end{array}$ & $\begin{array}{c}1 \\
0,02\end{array}$ & $\begin{array}{c}0,27 \\
0\end{array}$ & $\begin{array}{c}0,87 \\
0\end{array}$ & $\begin{array}{c}0,03 \\
0\end{array}$ \\
\hline 2340014 & $\begin{array}{l}\text { Phosphore total } \\
\mathrm{DBO}_{5}\end{array}$ & $\begin{array}{c}1 \\
0,54\end{array}$ & $\begin{array}{c}0,18 \\
0\end{array}$ & $\begin{array}{c}0,98 \\
0\end{array}$ & $\begin{array}{c}0,01 \\
0\end{array}$ \\
\hline 2340012 & $\begin{array}{l}\text { Phosphore total } \\
\mathrm{DBO}_{5}\end{array}$ & $\begin{array}{c}1 \\
0,29\end{array}$ & $\begin{array}{c}0,24 \\
0\end{array}$ & $\begin{array}{l}1 \\
0\end{array}$ & $\begin{array}{c}0,21 \\
0\end{array}$ \\
\hline
\end{tabular}

de 1994, les probabilités de dépassement vont en effet de 0,12 à 0,395 selon les points de contrôle. L'examen des résultats de l'été 1983 montre que, pendant toute cette période, les probabilités de dépassement à tous les points de contrôle sont très importantes même si l'on considère la situation de l'assainissement telle qu'elle était en 1994. Ainsi, le critère est dépassé pendant tout l'été à la station 2340012 (R94A83) et $98 \%$ des jours durant l'été à la station 2340014 (R94A83). Pour la chronique météorologique de 1994 (R94A94 et R83A94), ces deux probabilités diminuent à 0,01 pour la station 2340014 et 
0,21 à la station 2340012 (prise d'eau de Charny) ce qui montre le caractère très contraignant de l'été 1983.

Pour la $\mathrm{DBO}_{5}$, la situation est tout autre. Si les concentrations de ce polluant pouvaient dépasser à certaines occasions le critère avant la diminution des charges (simulations R83A83 et R83A94), ces probabilités sont ramenées à zéro si l'on considère la situation des rejets en 1994 et ce tant pendant la période estivale que pour toute l'année.

Les résultats montrent aussi que les concentrations d'azote totale sont toujours inférieures au critère utilisé pour les formes azote-nitrate et nitrite pour le traitement de l'eau brute et pour le contact primaire, à savoir $10 \mathrm{mg}-\mathrm{N} / \mathrm{L}$ (Ministère de l'Environnement et de la Faune, 1992). La valeur maximale enregistrée pour toutes les simulations, à tous les points de contrôle, est de 3,96 mg-N/L d'azote total et est obtenue lors de la simulation R83A83. Cette valeur est donc bien en deçà du critère précédent qui ne s'applique de plus qu'aux formes azote nitrate et nitrite. La médiane des concentrations en azote total à la station 2340012 (prise d'eau de Charny) pour la situation avant assainissement était de $0,86 \mathrm{mg}-\mathrm{N} / \mathrm{L}$ et est passée à $0,54 \mathrm{mg}-\mathrm{N} / \mathrm{L}$ après assainissement (chronique météorologique, 1983). A la lumière de ces résultats, il appert que les apports en azote d'origine urbaine ne sont donc pas des sources de contamination majeures.

Si l'on compare enfin les réductions obtenues pour les différentes variables selon que l'on se situe dans la partie amont ou aval du bassin, on note que ces réductions vont en croissant de l'aval vers l'amont. Ainsi, à titre d'exemple, pour le phosphore total, la probabilité annuelle de dépassement (tableau 8) passe de 0,55 (R83A83) à 0,19 (R94A83) à la station 234006 (Sud-Ouest de Saint-Ludger) alors que la même probabilité passe de 0,715 (R83A83) à 0,62 (R94A83) à la station la plus en aval (station 2340012). Ces résultats reflètent la distribution spatiale des pressions urbaines dans le bassin versant et l'importance de ces pressions dans les secteurs de la moyenne et basse Chaudière.

\section{6 - CONCLUSION}

La présente étude avait pour objectif de vérifier comment l'instauration du programme d'assainissement des rejets urbains sur le bassin versant de la rivière Chaudière s'est traduite en matière de réduction des concentrations au cours d'eau, et donc en probabilité de dépassement de différents critères de qualité de l'eau. L'utilisation du système de modélisation intégrée GIBSI dans un tel contexte permet la prise en compte des facteurs hydrobiochimiques déterminant ces concentrations. Il est dès lors possible d'estimer, pour chaque tronçon formant le bassin versant, les concentrations journalières des principales variables de qualité de l'eau. Deux scénarios ont été considérés pour décrire la situation de l'assainissement urbain, un premier correspondant à l'année 1983, représentatif de la période initiale d'instauration du programme d'assainissement, et un deuxième correspondant à l'année 1994 pour lequel une bonne partie du programme était déjà en place. Deux chroniques météoro- 
logiques ont été retenues pour chacun de ces scénarios, à savoir celles des années 1983 et 1994. La comparaison s'est effectuée en quatre points de contrôle situés aux quatre stations principales de mesures de la qualité de l'eau sur le bassin versant. Les simulations effectuées ne considèrent que les apports ponctuels d'origine urbaine. Les rejets industriels non raccordés à un réseau municipal et les apports diffus d'origines urbaine et agricole sont négligés. Pour fin d'analyse, les résultats ont été comparés à certains critères de qualité de l'eau afin de voir dans quelle mesure le programme d'assainissement a permis de réduire la probabilité de dépassement de ces critères aux points de contrôle.

En matière de charges rejetées au cours d'eau dans les différents sousbassins, les réductions sont importantes entre les deux périodes considérées. Pour le bassin versant de la Chaudière, ces réductions sont de l'ordre de $37 \%$ pour l'azote total, de $38 \%$ pour le phosphore total et de $83 \%$ pour la $\mathrm{DBO}_{5}$. Dans tous les cas, l'année 1986 marque en quelque sorte un tournant pour lequel les charges urbaines subissent d'importantes diminutions à l'échelle des sous-bassins considérés.

Les résultats montrent que les réductions des charges en azote total et en $\mathrm{DBO}_{5}$ ont entraîné des réductions significatives des concentrations au cours d'eau. Toutefois, les charges rejetées au cours d'eau avant l'instauration du programme d'assainissement n'entraînaient pas de dépassement important des critères pour ces deux variables. Dans le cas plus spécifique de l'azote total, les concentrations obtenues sont toujours inférieures au critère de $10 \mathrm{mg}-\mathrm{N} / \mathrm{L}$. Pour la $\mathrm{DBO}_{5}$, la réduction des charges a permis un respect du critère de $3 \mathrm{mg} / \mathrm{L}$ pour les deux chroniques météorologiques considérées.

Pour le phosphore total, la situation est tout autre. La probabilité de dépassement du critère retenu reste très importante même dans le contexte actuel de l'assainissement urbain. Ce résultat suggère que la dimension urbaine, même pour un bassin aussi faiblement urbanisé que celui de la rivière Chaudière, doit être prise en compte dans la problématique actuelle du phosphore, et ce même si d'importants efforts au niveau de l'assainissement ont été réalisés au chapitre du traitement du phosphore.

Les résultats et conclusions présentés dans cet article reposent sur une validation du système GIBSI s'appuyant sur l'utilisation de valeurs vraisemblables de paramètres et sur la base d'hypothèses au sujet de certaines variables d'entrée des modèles pour lesquels nous ne disposons que d'une information globale et approximative comme, par exemple, les données relatives aux sources diffuses de pollution. Par ailleurs, les sources diffuses d'origine urbaine, les possibles débordements de réseau unitaire et les apports " naturels" non anthropiques de polluants ne sont pas considérés et contribuent certainement, dans certaines situations et en certains points du réseau hydrographique à tout le moins, à la qualité de l'eau en rivière. La prise en compte de l'ensemble de ces sources de polluants et de leurs variabilités spatiale et temporelle à l'échelle d'un bassin versant est, on le devine, bien au-delà de la caractérisation actuellement disponible. Dans un tel contexte, certaines hypothèses réalistes doivent être formulées afin de permettre une estimation des charges rejetées au cours d'eau. Le calage des paramètres du modèle de qualité de l'eau est donc difficile puisqu'il est tributaire de ces hypothèses et des incertitudes relatives aux données d'entrée. Cette situation, combinée au 
fait que peu de données de qualité soient disponibles, nous a conduit à sélectionner un ensemble de valeurs de paramètres réaliste conforme aux valeurs généralement rapportées. Une analyse d'incertitudes devra cependant être conduite afin de voir l'impact des incertitudes sur ces valeurs de paramètres sur les résultats de simulation et de voir dans quelle mesure ces incertitudes peuvent modifier les conclusions de la présente étude. Des travaux sont actuellement réalisés en ce sens.

Le présent travail constitue le premier jalon dans une démarche visant à caractériser de façon plus complète les contributions provenant des différentes sources de polluants à l'échelle d'un bassin versant. Une comparaison des concentrations simulées en ne considérant que les apports urbains avec les données observées permettra de mieux apprécier les contributions d'origines ponctuelle et diffuse selon le régime hydrologique. Ainsi, en période d'étiage, les concentrations simulées sur la base des seules contributions urbaines devraient se comparer aux valeurs mesurées puisque les contributions diffuses sont généralement plus faibles dans ce cas. La situation inverse devrait apparaître en périodes de crue. Une meilleure appréciation des contributions d'origine diffuse et des conditions pour lesquelles celles-ci sont importantes devrait aussi nous permettre de mieux les caractériser et de valider certaines hypothèses utilisées pour les estimer.

\section{REMERCIEMENTS}

Les auteurs remercient sincèrement MM. Michel ROUX et Daniel LEMIEUX, respectivement technicien en informatique et stagiaire à l'INRS-Eau, pour leur assistance technique. De plus, ils tiennent à exprimer leur reconnaissance aux réviseurs pour leurs questions et commentaires judicieux qui ont permis d'améliorer substantiellement la qualité de cet article.

\section{RÉFÉRENCES BIBLIOGRAPHIQUES}

ARNOLD J.G., WILLIAMS J.R., 1995. SWRRB - A Watershed Scale Model for Soil and Water Resources Management. In: SINGH V.P. (ed.), Computer Models of Watershed Hydrology, Water Resources Publications, Highlands Ranch (CO), 847-908.

BOWIE G.L., MILLS W.B, PORCELLA D.B., CAMPBELL C.L., PAGENKOPF J.R., RUPP G.L., JOHNSON K.M., CHAN P.W.H., GHERINI S.A., CHAMBERLIN C.E., 1985. Rates, constants, and kine- tics formulations in surface water quality modeling (second edition). Rep. EPA/600 600/ 3-85/ 040, U.S. EPA, Athens, Ga., 1985, 455 p.

BRIËE F.G., 1997. Distribution et collecte des eaux, édition revue et augmentée ; Éditions de l'École Polytechnique de Montréal, Montréal.

BROWN L.C., BARNWELL Jr. T.O., 1987. The enhanced stream water quality models QUAL2E and QUAL2E-UNCAS: Documentation and user manual. Rep. 
EPA/600/3-87/007, US EPA, Athens, Ga., 1987, 141.

FORTIN J.P., MOUSSA R., BOCQUILLON C., VILLENEUVE J.P., 1995. HYDROTEL, un modèle hydrologique distribué pouvant bénéficier des données fournies par la télédétection et les systèmes d'information géographique. Rev. Sci. Eau, 8, $97-124$.

FORTIN J.P., TURCOTTE R., MASSICOTTE S., MOUSSA R., FITZBACK J., 2001a. A distributed watershed model compatible with remote sensing and GIS data, part 1: Description of the model. J. Hydrol. Engng., ASCE 6 (2), 91-99.

FORTIN J.P., TURCOTTE R., MASSICOTTE S., MOUSSA R., FITZBACK J., 2001b. A distributed watershed model compatible with remote sensing and GIS data, part 2: Application to the Chaudière watershed. J. Hydrol. Engng., ASCE 6 (2), 100-108.

GANGBAZO G., 1997. Contrôle de la pollution diffuse agricole par l'approche des objectifs environnementaux de rejet. Vecteur Environnement, 30 (4), 25-31.

GANGBAZO G., PAINCHAUD J., 1999. Incidence des politiques et programmes d'assainissement agricole sur la qualité de l'eau de six rivières, 1988-1995. Vecteur Environnement, 32 (1), 29-36.

MAILHOT A., ROUSSEAU A.N., MASSICOTTE S., DUPONT J., VILLENEUVE J.P., 1997. A watershed-based system for the integrated management of surface water quality; the GIBSI system. Water Sci. and Technol., 36 (5), 381-387.

MARANDA Y., SASSEVILLE J.L., 1999. Efficacité de l'assainissement des eaux usées sur le bassin de la rivière Chaudière (Québec, Canada). Rev. Sci. Eau, 12 (3), 485-507.

Ministère de l'Environnement et de la Faune du Québec (MEF), 1992. Critères de qualité de l'eau (1990, révision 1992). Service de l'évaluation des rejets toxiques et Direction de la qualité des cours d'eaux, $425 \mathrm{p}$.

Ministère de l'Environnement et de la Faune du Québec (MEF), 1997. Rapport d'évaluation des ouvrages municipaux d'assainissement des eaux du PAEQ. Direction des politiques du secteur municipal, Service de l'assainissement des eaux de consommation, mars 1997, $52 \mathrm{p}$.
NOVOTNY V., CHESTERS G., 1981. Handbook of nonpoint pollution, Van Nostrand Reinhold Environmental Series, Litton Educational Publishinng Inc., 555.

RENARD K.G., FOSTER G.R., WEESIES G.A., MCLOOD D.K., YODER D.C., 1997. Predicting Soil Erosion by Water: A Guide to Conservation Planning with the Revised Universal Soil Loss Equation (RUSLE). Agricultural Handbook 703, US Department of Agriculture, Agricultural Reserach Service, Washington DC, $404 \mathrm{p}$.

ROUSSEAU A.N., MAILHOT A., GARIÉPY S., SALVANO E., VILLENEUVE J.P., 2002. Calcul de probabilités de dépassement d'objectifs environnementaux de rejets de sources ponctuelle et diffuse à l'aide du système de modélisation intégrée GIBSI. Rev. Sci. Eau, $15 / \mathrm{n}^{\circ}$ spécial.

ROUSSEAU A.N., MAILHOT A., TURCOTTE R., DUCHEMIN M., BLANCHETTE C., ROUX M., DUPONT J., VILLENEUVE J.P., 2000. GIBSI: an integrated modelling system prototype for river basin management. Hydrobiologia, 422-423, 465-475.

SIMONEAU M., 1998. Le bassin de la rivière Chaudière : qualité des eaux, 1979-1996. In: Ministère de l'Environnement et de la Faune (ed.), Le bassin de la rivière Chaudière : l'état de l'Écosystème aquatique, 1996, 2.1-2.49.

SIMONEAU M., PELLETIER L., MARTEL N., 1998. Le bassin de la rivière Chaudière : profil géographique, sources de pollution et interventions d'assainissement. In: Ministère de l'Environnement et de la Faune (ed.), Le bassin de la rivière Chaudière : l'état de l'Écosystème aquatique, 1996, 1.1-1.34.

SMITH D.I., 1978. Water Quality for RiverReservoir Systems. Resources Management Associates inc. Lafayette, Californie. 210.

TCHOBANOGLOUS G., SCHOEDER E.D., 1985. Water Quality, Addison-Wesley (ed.), $768 \mathrm{p}$.

TURCOTTE R., ROUSSEAU A.N., MAILHOT A., VILLENEUVE J.P., DUPONT J., 1999. GIBSI: an integrated modelling system for sustainable watershed management. Proceedings of the Environmental Permitting Symposium, Air \& Waste Management Association (AWMA) Symposium 
\& Courses, Research Triangle Park, NC, 17-19 février 1999. One Gateway Center, Third Floor, Pittsburgh, PA 15222.

VILLENEUVE J.P., BEAUCHEMIN J., BOLDUC P., DROUOT C., DUCHEMIN M., GAGNON J.F., MAILHOT A., ROUSSEAU A.N., TREMBLAY J.F., TURCOTTE R., 1997. Rapport d'étape du projet GIBSI : Gestion intégrée des bassins versants à l'aide d'un système informatisé. INRS-Eau, Ste-Foy, Québec, Canada, Rapport d'étape no $R-462 b$.

VILLENEUVE J.P., BLANCHETTE C., DUCHEMIN M., ETONG N., MAHEU D. MAILHOT A., MARTIN A., ROUSSEAU A.N., ROUX M., TRÉPANIER S., TURCOTTE R., 1998. Rapport synthèse du projet GIBSI : Gestion intégrée des bassins versants à l'aide d'un système informatisé. INRS-Eau, Ste-Foy, Québec,
Canada, Rapport Scientifique no RS462.

WES (Waterways Experiment Station), 1982. CE-QUAL-R1: A Numerical One-Dimensional Model of Reservoir Water Quality, Users Manual. Environemental and Water Quality Operational Studies (EWQOS), US Army Corps of Engineers, Waterways Experiment Station, Vicksburg, Missisipi, États-Unis.

WILLIAMS J.R., 1995. The EPIC model. In: SINGH V.P. (ed.), Computer Models of Watershed Hydrology, Water Resources Publications, Highlands Ranch (CO), 909-1000.

WISCHMEIER J.R., SMITH D.D., 1978. Predicting rainfall erosion losses - A guide to conservation planning. Agricultural Handbook $n^{\circ} 537$, US Department of Agriculture, Washington DC, 58 p. 